\title{
Comparative Analysis Study on SSD, HDD, and SSHD
}

\author{
Ms.Hepisuthar ${ }^{a}$, Dr. Priyankasharma ${ }^{b}$ \\ ${ }^{a}$ Department Of Research and Publication divisionRashtriyaraksha university Gandhinagar \\ ${ }^{\mathrm{b}}$ Department of Research and Publication Division, RashtriyaRaksha University Gandhinagar
}

Article History: Received: 10 November 2020; Revised 12 January 2021 Accepted: 27 January 2021; Published online: 5 April 2021

\begin{abstract}
In the Current Century, permeant storage devices and methods of storing data changed from traditional HDD to SDD. In this document, we discuss the merge of HDD and SSD. The Abbreviation of SSHD is called the solid-state hybrid disk. A mixture of both secondary devices to enhance the performance of the system. Inside the SSD, data movement events occur without any user input. Recent research has suggested that SSD has only the Replacement of secondary storage. HDD is also good in life span with longer life. It's more reliable for long time data contained in this. HDD storage has typical magnetic fields for store data. SSD contains NAND flash memory to write the data in the drive. Based on the method and material of storing different. HDD and SSD feature well to upgrade with technology in Computer filed. For enhancing computing speed and excellent processing SSHD good to use in computer.Ratio increase of SSHD usage in current laptop and in computer system.
\end{abstract}

Keywords: SSD, Secondary Storage Device, NAND Flash Memory, HDD, SSHD, Hybrid Drive

\section{Introduction}

As the design of computers has improved with time, many manufacturers have moved from traditional HDDs to SSDs. These SSDs are tiny and more compact, thin in size than HDDs. They are also more robust and Highspeed data transfer rate and resilient to vibration and allow for much greater Input/ Output (I/O) data transfer speeds. These drives contain no moving parts and store each bit of data in a floating gate transistor rather than on a magnetic spinning platter of an HDD. Although these new types of drives have many advantages, they also have some limitations and unexpected output. These drives have a limited number of writes per cell, can only write in pages, and must erase a full block of pages before rewriting any single page. Depending on the type of NAND flash memory module, some SSDs may come with several bad areas (which means a bad location where data cannot store) that need to be corrected. Because of these noted limitations, data is stored using non-traditional methods such as using error correction code, bad area management, and scrambling, etc.

SSD is a solid-state gadget that utilizes an incorporated circuit get together with the end goal of information stockpiling. It doesn't include any moving parts like its partner the HDD, henceforth it is known as a solid-state drive. [1,4] SSD depends on Flash memory. SSD is certifiably not an innovative advancement of a hard plate, it is to be sure an innovation that mirrors the conduct of the HDD. In (Figure 1.)Shows the pictorial representation of a 250 GB capacity internal storage Samsung SSD. Many more companies also release different variants of storage capacity drives. SSD much famous within a short time because of the speed of access data from NAND flash memory. SSD control by the controller in like Master and many small slaves (Flash-based Memory module). The controller handles each small block of flash NAND. [11, 12] Nowadays SSD comes by default in the computer system. SSD gives batter throughput in performance.

\section{SSD Components}

SSD is comprised of two main parts - the Controller and the Flash memory, along with a few other components that are set on a Printed Circuit Board (PCB), which is secured inside a case (Figure 1). All the modules of SSD going to cover in detail below. 


\section{Method and Material}

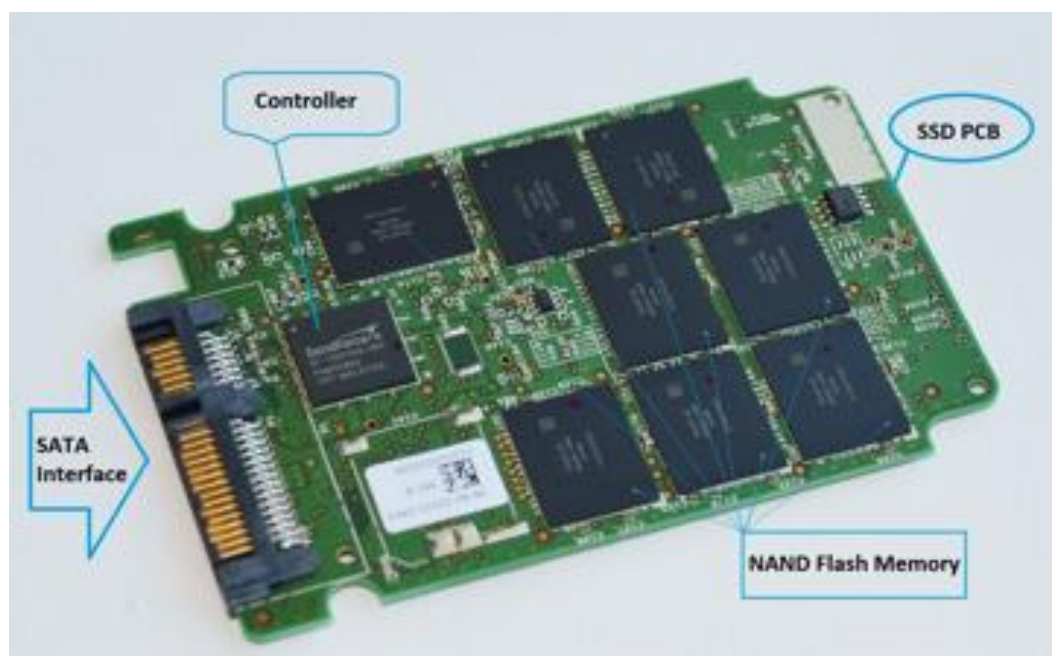

Figure. 1 Solid State Drive Hardware PCB

\section{Controller}

A Controller is an installed processor that connects the Flash memory parts to the info and yield interfaces. It executes the codes given by the gadget's firmware (unchangeable programming modified into a read-just memory (ROM)). It contains the chip, blunder remedy, support, and glimmer interface modules.[7] The controller can choose how the SSD would complete its highlights, for example, perusing, composing, mistake checking, trash assortment, deleting, wear leveling, and so forth. It plays out the read and composes tasks everywhere throughout the memory chip. In current SSDs, extra stockpiling is accessible to the controller. SSD controller has appeared in (Figure 2).

\section{Flash Memory}

The flash memory is a non-unpredictable memory - that is, it holds memory after the force is expelled, and it erases information at the square level. Information put away instantly memory must be eradicated first, before any information is changed into those recollections. Gadgets utilizing streak memory clear out Information at the square level, and they rework information at the byte level or numerous byte page level. SSD Memory utilizes NAND or NOR innovation. NOR Flash chips are increasingly slow constrained to modify perseverance ability; in this manner, NAND is the better contribution utilized in the SSD [3]. Flash memory in SSD appears in Figure 2.

\section{NAND Flash Memory}

In NAND streak memory, the essential unit is the phone, each putting away the slightest bit, speaking to either 0 or 1 . These phones are associated with infinitesimally in an arrangement and help decrease the physical size by just having one electrical connector between them.[3] NAND streak memory performs successive access on code regions and has a higher stockpiling limit. It performs quick read, compose, and eradicate when contrasted with NOR memory and has a delete square range from $8 \mathrm{~K}$ bytes to $32 \mathrm{~K}$ bytes. NAND goes about like a circle drive as it likewise has sequential memory where information is tended to and put away in squares. NAND-based SSD's are intended to copy a turning attractive plate offering quicker access time. NAND comes in single-layer and multilayer cells. While venture SSD utilizes a solitary layered NAND, $[5,10,11]$ which is quicker and costly, customer grade SSD utilizes multilayer NAND, which is slower however monetary. There are currently five types of NAND flash memory in the market. The difference lies in the number of bits that each cell can store. The data bits that can be stored in each unit of SLC NAND, MLC, TLC, QLC and PLC are one, two, three, four and five bits respectively. Therefore, each cell of SLC NAND can store a "0" or "1", and each cell of MLC NAND can store "00", "01", "10" or "11", and so on. These five types of NAND provide different levels of performance and durability with different pricing ranges. Among them, SLC is the higher-performance and most expensive type in the NAND market.

\section{SATA Interface}

SATA (Serial Advanced Technology Attachment) is a computer bus especially for connecting mass storage devices to host systems. This interface was designed to be a much faster interface with a higher data rate on serial paired lines for transmitting and receiving data. SATA interface is shown in Figure 1. 


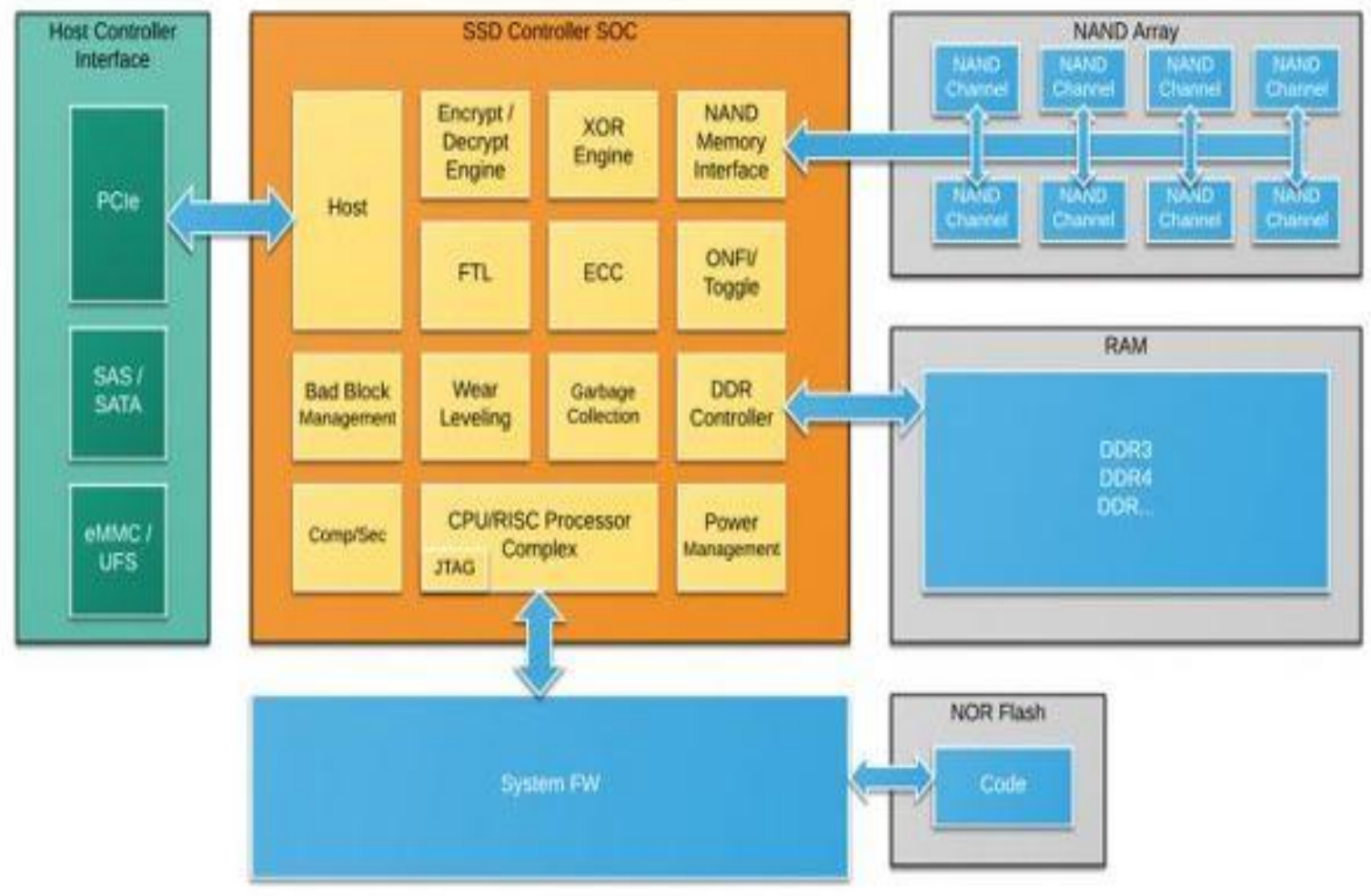

Figure 2. SSD Internal Structure Block Diagram

SSD Concepts

Like any other storage media, SSD also has various functions like erasing, rewriting, etc., and processes like TRIM, Garbage Collection, and Wear Leveling. These are as described next.

\section{TRIM}

TRIM is a method of data removal in the SSD. The TRIM function deletes the data blocks that are marked as 'deleted'. TRIM is an ATA command, which serves as a communication medium between the file level and the block level where it provides information to the SSD about the deleted files and will alert it to mark those pages as stale. Reads and writes damage the flash memory, but because of the TRIM command, there is a lower rate of data being written on the drive as it informs the SSD about skipping the process of rewriting data until the next block is erased. This helps in increasing the life span of the SSD. TRIM,[8] along with garbage collection and the wearleveling function, work together to increase the lifetime of the SSD.

\section{Garbage Collection}

It is one of the fundamental processes in SSD as NAND Flash-based devices cannot overwrite data that is already there and must go through an erase cycle. SSD first copies the data and writes it to empty pages of a different block.[6]Then the cells of the new block are erased and new data is written. This is Garbage Collection. It is a background process, which works as a housekeeping service.[8]

\section{Wear Leveling}

NAND flash has individually erasable all the blocks, each of which can be put through a finite number of erase cycles before becoming unreliable. That is, after a specific number of cycles for each block, the error rate can significantly increase. Unfortunately, in most cases, flash media is not used evenly.[16] In certain areas, such as for the file system, the data is updated more frequently than for other areas and will wear out sooner in those areas. Wear leveling mitigates this issue by arranging data so that erasures and re-writes are distributed evenly across the entire device. Thus, no single sector prematurely fails due to a high concentration of program/erase cycles. Foreman uses an advanced wear-leveling algorithm, which can efficiently spread usage throughout the entire flash media area. By implementing both dynamic and static wear-leveling algorithms, the life expectancy of the flash media can be improved significantly. Wear leveling is a life-span protective technique that ensures that certain NAND blocks are not written or erased more often than the other blocks. Manufacturers employ this 
technique to increase the life of their product and counteract the degradation of the NAND flash. [16] Wear leveling ensures balanced data distribution over the physical cells. Flash memory in SSD allows only a certain number of reading and writing processes - generally ranging from 10,000 to 100,000 cycles.[14]

1. Dynamic wear leveling: Here the blocks that undergo rewriting are repositioned to new blocks. The controller tracks the write/erase cycles for all blocks and selects one with the least number of write/erase cycles endured. It addresses the issue of repeated writes to the same block by redirecting new writes to a different physical block, avoiding early wear out of the frequently used blocks. Dynamic wear leveling cannot guarantee even leveling as there may be data in the drive that may have remained unchanged for a long period. In such cases, the active, frequently used blocks will undergo wear leveling and the dormant ones may be untouched.

2. Static wear leveling: Contrary to dynamic wear leveling, all data blocks are evenly distributed and leveled including those whose data is not to be written. To evenly distribute data blocks the controller selects blocks from the static data pool with the lowest program/ erase count and that block is swapped with the block in the free data pool with the highest program/erase count.

This is resolved by Static wear leveling, which includes the static data blocks. However, moving static data will take time and energy and will also cost more program/erase cycles.

\section{SSD Wear Leveling Algorithms}

Although SLC NAND Flash memory provides a high level of performance, it still has a large lifespan. There are so many times a program and erase cycle can occur before a failure takes place and data is put at risk. To ensure that SLC memory lasts for as long as possible, SSDs rely on wear-leveling algorithms. These algorithms ensure that writes are evenly distributed throughout the SSD so that blocks do not wear out prematurely. To further explain SSD wear-leveling algorithms here are the main concepts.[6,8]

\section{Non-Sequential Writing}

In a perfect world, when a host system provides an LBA for data it wants to read or write, the SSD controller would read or write the LBA to the same physical block address. In reality, this doesn't happen for many different reasons, including.

- $\quad$ File system directories that are not updated after files are changed.

- Mismatches in data sizes between the host and block sizes of the SSD's NAND.

- The process of reclaiming blocks that have been previously written.

These are just a few of the factors that lead to non-sequential writing. Without any intervention, this could cause the NAND to use the maximum allowable number of endurance cycles for certain cells, without using other cells at all.

\section{Wear Leveling and Endurance Cycle Balancing}

Wear leveling algorithms that are used in SSD controllers address the impacts of non-sequential writing to maximize the efficiency of the SSD. There is no single method of wear leveling that is used across all SSDs, as designers often use their versions. Some algorithms store host data for LBAs to a place with the smallest amount of used endurance cycles. Others move static data to different physical locations in the NAND so that the cells that previously held static data can be used for their maximum number of endurance cycles. Typically, SSD wears leveling algorithms work as part of a multifaceted approach for managing SSD endurance.

\section{Overprovisioning}

This is the extra storage capacity that is included in a solid-state drive (SSD). SSD overprovisioning can increase the endurance of an SSD by distributing the total number of write and erases across a larger population of NAND flash blocks and pages over time.

Maintaining the integrity of SSDs due to garbage collection, secure delete, wear leveling, and data remapping is a serious issue that makes it more difficult and challenging for the forensics investigator to make the digital evidence accepted in a court of law since the hash values of the evidence keeps changing with time.

\section{Solid State Hybrid Drive}

With solid-state hybrid drive technology, a conventional hard drive is expanded to include faster flash memory. A special algorithm recognizes frequently used files and moves them to the flash memory of the drive, thus the access time and the reading speed of this frequently used data can be reduced considerably. As of Windows Vista, the operating system itself provides such an algorithm so that an SSHD can also be created from an SSD and an HDD, for example. 

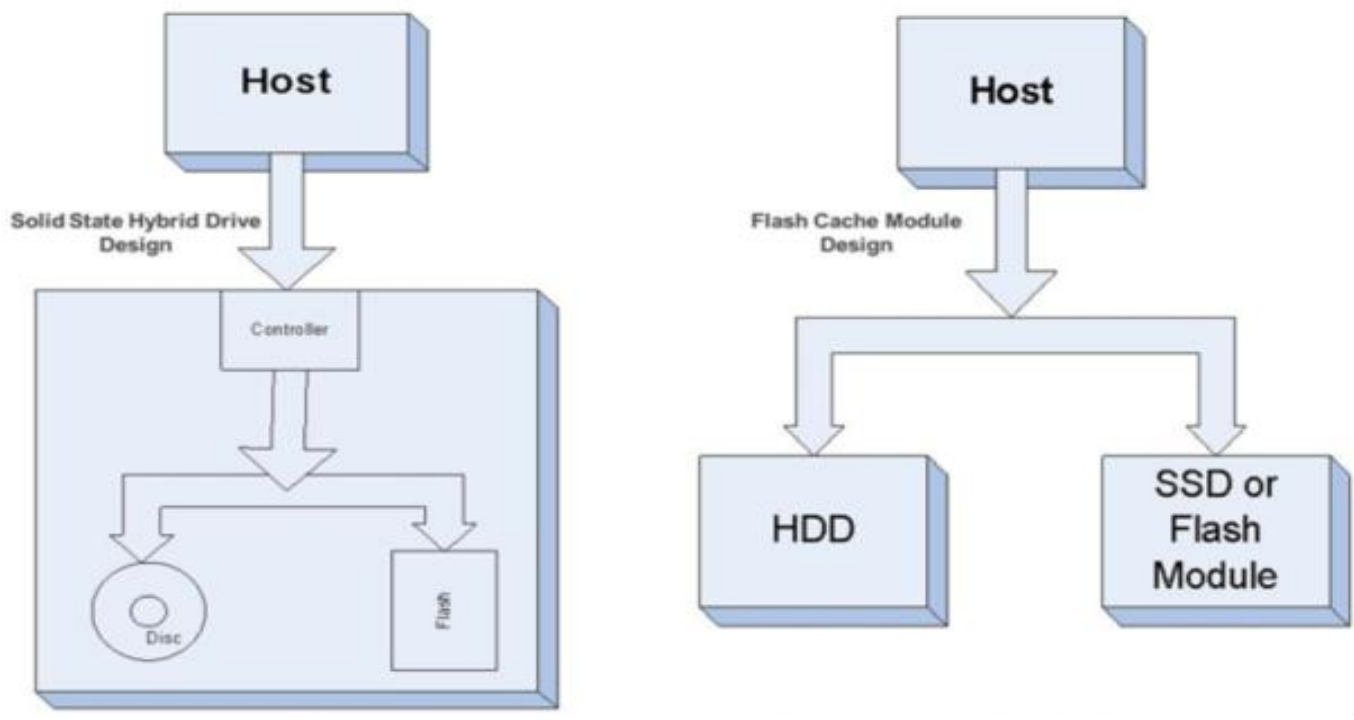

Figure 3. Hybrid Drive

The algorithm that is used in the Seagate laptop SSHD, on the other hand, is made available directly from the hard drive, whereby the advantage of the additional flash memory comes into play even with older operating systems such as Windows XP or even Linux file systems. Seagate calls this technology Adaptive Memory Technology. The adaptive memory technology is anchored in the firmware of the SSHD and can therefore also be expanded with updates and improvements. The speed of the SSHD is therefore primarily linked to the usage needs of the user. Thanks to the Adaptive Memory Technology developed by Seagate, SSHDs are said to be around 4.5 times faster than conventional magnetic disk hard drives. However, this added value of the SSHD requires a certain period of getting used to before the SSHD could "learn" which of the user data is important for the user, which is requested frequently and should therefore be available in the flash memory.

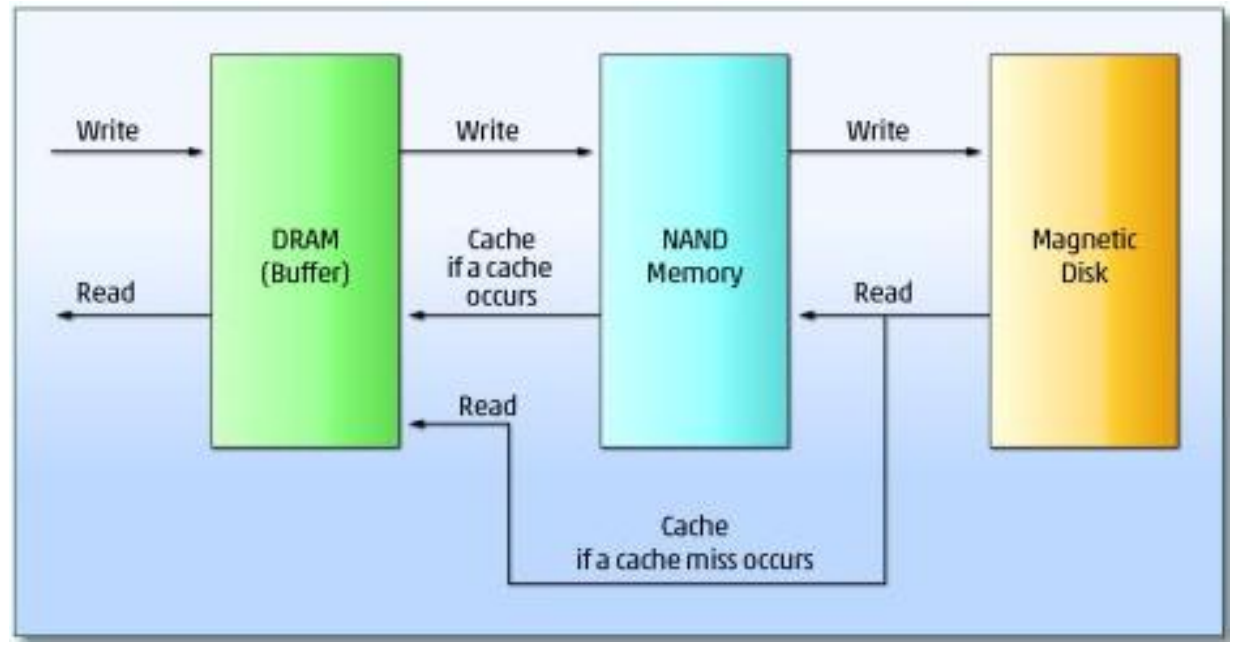

Figure 4. Design Concept of Hybrid Disk drive

However, this "learning speck" is a not insignificant problem for our classic test of a hard drive or SSD. Many benchmarks aim at a certain routine being carried out once or even a file is created and the benchmark "running" in this file. Even if individual benchmarks are repeated several times, it cannot be guaranteed with certainty that the test will be carried out in the flash memory on the hard drive at some point. Especially not if the benchmark regenerates the file with each run. Besides, the Seagate laptop SSHD's flash memory is used solely for reading files. Copying or writing a file can therefore only be accelerated to a limited extent by Adaptive Memory Technology, namely when the same file is constantly being copied, which is not exactly relevant in practice.

\section{Results and discussion}

If a comparative study with HDD and SSD it will describe in the form of difference. SSD is next-generation Secondary storage but also more reliable to use and replace HDD. SSD provides batter competition to HDD in all 
parameters. Most of the factors were good compare to HDD. SSD life-limited and also recovery of data it's a little hard than HDD. In the case of Speed and performance SSD more efficient and powerful. [4,9]

Table 1. Comparison table of SSHD, SSD, HDD

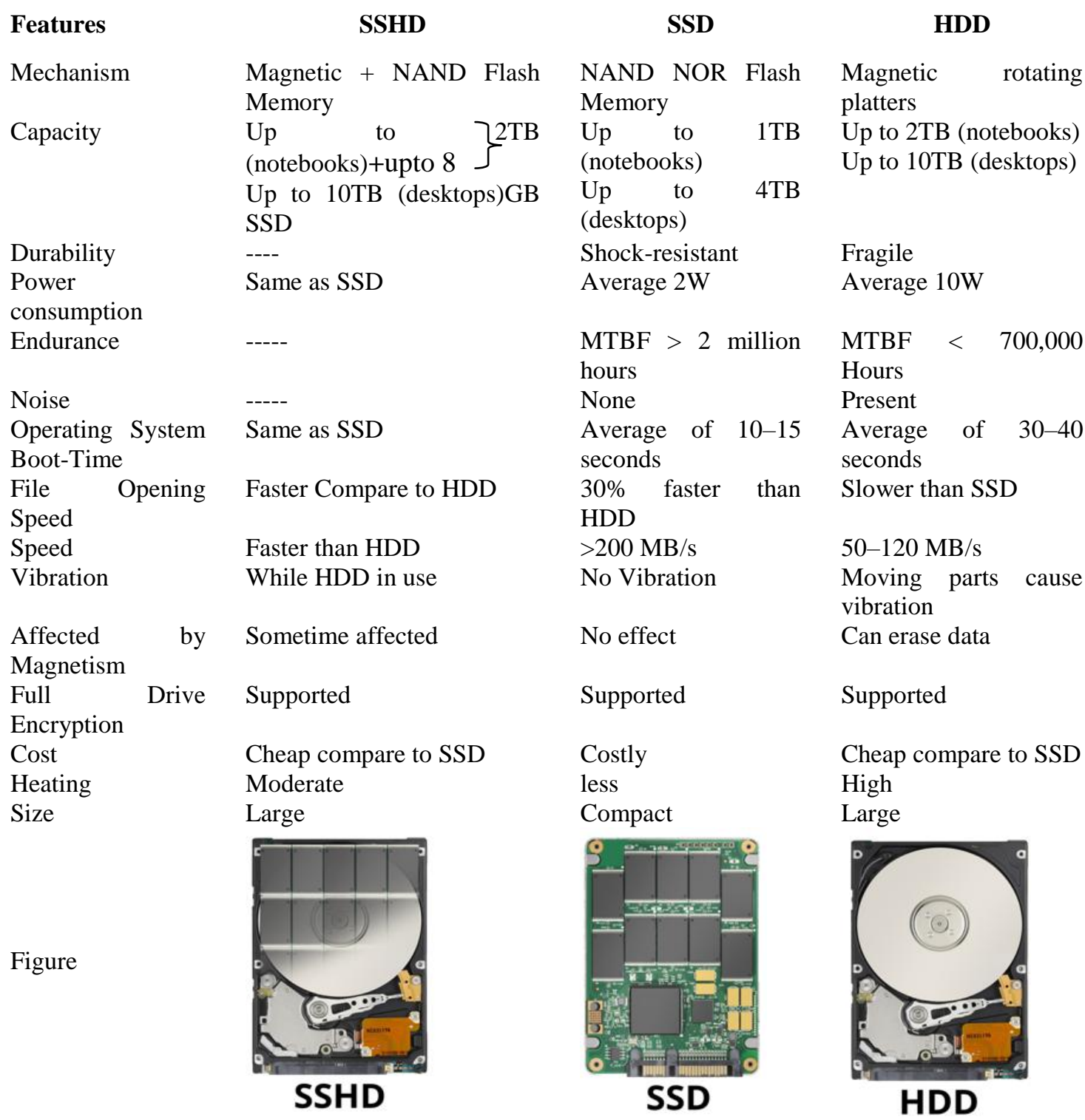

Most of all device contains some benefits and some lacking inside any hardware module so here are discussed SSD's good and bad points.[12,13]

\section{Conclusions}

Based on the upcoming generation people want both speed processing CPU power and a good life span for the long live data.so now a new concept release in Information technology called SSHD. SSHD abbreviation for Solid State Hybrid Drive.Combination of both HHD and SSD. It's more usable nowadays. It's the most reliable and easy to use on all devices.

\section{Acknowledgments}

This paper and the research behind it would not have been possible without the exceptional support of my Supervisor, Dr. Priyanka Sharma. Her Enthusiasm, Knowledge, and exacting of details have been an inspiration and kept my work on track from my first encounter with the survey paper. I am also thankful to RashtriyaRaksha University, Gandhinagar.

\section{Abbreviation \\ HDD - Hard Disk Drive}


SSD - Solid State Drive

SSHD - Solid State Hybrid Disk

PCB - Printed Circuit board

SLC - Single Level Cell

LBA - Logical Block Address

SOC - System on Chip

\section{References}

Chang, L. On Efficient Wear Leveling for Large Scale Flash Memory Storage Systems. SACee 07 March 11-15, 2007, Seoul, Korea.

Micron technology, "Wear Leveling Techniques in NAND Flash,” Tech. Rep. (2008)

Takeuchi, K. (2009). Novel Co-Design of NAND Flash Memory and NAND Flash Controller Circuits for Sub30 nm Low-Power High-Speed Solid-State Drives (SSD). IEEE Journal of Solid-State Circuits, 44(4), 12271234.

Rizvi, S. and Chung, T. (2010). Flash SSD vs. HDD: High performance oriented modern embedded and multimedia storage systems, 2010, 2nd. International Conference on Computer Engineering and Technology.

R. Micheloni, A. Marelli, and S. Commodaro, "Nand overview: from memory to systems," in Inside NAND flash memories. Springer, 2010, pp. 19-53.

J. Lee, Y. Kim, G. M. Shipman, S. Oral, and J. Kim, Preemptible i/o scheduling of garbage Collection for solid state drives, Computer- Aided Design of Integrated Circuits and Systems, IEEE Transactions on, vol. 32, no. 2, pp. 247260, 2013

J. Kim, Y. Lee, K. Lee, T. Jung, D. Volokhov, and K. Yim, "Vulnerability to flash controller for secure usb drives.” J. Internet Serv. Inf. Secure, vol. 3, no. 3/4, pp. 136-145, 2013.

Gubanov, Y., Afonin, O.: Recovering evidence from SSD drives: understanding TRIM, garbage collection and exclusions, Belkasoft, Menlo Park (2014)

Geier, F.: The differences between SSD and HDD technology regarding forensic investigations, Sweden (2015)

J. Cha, W. Kang, J. Chung, K. Park and S. Kang, "A New Accelerated Endurance Test for Terabit NAND Flash Memory Using Interference Effect," IEEE Transactions on Semiconductor Manufacturing, vol. 28, no. 3, pp. 399-407, Aug. 2015

D. Chang, W. Lin and H. Chen, "FastRead: Improving Read Performance for Multilevel-Cell Flash Memory," in IEEE Transactions on Very Large Scale Integration (VLSI) Systems, vol. 24, no. 9, pp. 2998-3002, Sept. 2016.

N .Y. Ahn and D.H. Lee, "Duty to Delete on Non-Volatile Memory," https://arxiv.org/abs/1707.02842 July 2017.

M. Kang, W. Lee and S. Kim, "Subpage-Aware Solid State Drive for Improving Lifetime and Performance," IEEE Transactions on Computers, vol. 67, no. 10, pp. 1492-1505, 1 Oct. 2018.

P. Wang et al., "Three-Dimensional NAND Flash for Vector-Matrix Multiplication," IEEE Transactions on Very Large Scale Integration (VLSI) Systems, vol. 27, no. 4, pp. 988-991, April 2019.

J. Ko et al., "Variation-Tolerant WL Driving Scheme for High-Capacity NAND Flash Memory," IEEE Transactions on Very Large Scale Integration (VLSI) Systems, vol. 27, no. 8, pp. 1828-1839, Aug. 2019.

SLC, MLC or TLC NAND for Solid State Drives by Speed Guide.net. https://www. speedguide.net/faq/slc-mlcor-tlc-Nand-for-solid-state-drives-406. Accessed 1 June 2020

NAND Bad Columns analysis and removal by ruSolute.http://rusolut.com/nand-badcolumns-analysis-andremoval/. Accessed 1 July 2020 